

\title{
Derivative Spectroscopy and the Continuous Relaxation Spectrum.
}

\author{
A.R. Davies ${ }^{\mathrm{a}, *}$, R. S. Anderssen ${ }^{\mathrm{b}}$, F. R. de Hoog ${ }^{\mathrm{b}}$, N. J. Goulding ${ }^{\mathrm{c}}$ \\ ${ }^{a}$ School of Mathematics, Cardiff University, Senghennydd Road, Cardiff CF24 4AG, UK. \\ Electronic mail: DaviesR@cardiff.ac.uk \\ ${ }^{b}$ CSIRO, GPO Box 664, Canberra, ACT 2601, Australia. Electronic mail: \\ Bob.Anderssen@csiro.au,Frank.deHoog@csiro.au \\ ${ }^{c}$ School of Earth Sciences, University of Bristol, University Road, Clifton, Bristol BS8 1SS, \\ UK. Electronic mail: n.goulding@bristol.ac.uk
}

\begin{abstract}
Derivative spectroscopy is conventionally understood to be a collection of techniques for extracting fine structure from spectroscopic data by means of numerical differentiation. In this paper we extend the conventional interpretation of derivative spectroscopy with a view to recovering the continuous relaxation spectrum of a viscoelastic material from oscillatory shear data. To achieve this, the term "spectroscopic data" is allowed to include spectral data which have been severely broadened by the action of a strong low-pass filter. Consequently, a higher order of differentiation than is usually encountered in conventional derivative spectroscopy is required. However, by establishing a link between derivative spectroscopy and wavelet decomposition, high-order differentiation of oscillatory shear data can be achieved using specially constructed wavelet smoothing. This method of recovery is justified when the reciprocal of the Fourier transform of the filter function (convolution kernel) is an entire function, and is particularly powerful when the associated Maclaurin series converges rapidly. All derivatives are expressed algebraically in terms of scaling functions and wavelets of different scales, and the recovered relaxation spectrum is expressible in analytic form. An important feature of the method is that it facilitates local recovery of the spectrum, and is therefore appropriate for real scenarios where the oscillatory shear data is only available for a finite range of frequencies. We validate the method using synthetic data, but also demonstrate its use on real experimental data.
\end{abstract}

Keywords: continuous relaxation spectrum, wavelet smoothing, weighted Gegenbauer wavelets, delta sequences.

${ }^{*}$ Corresponding author. Electronic mail: DaviesR@cardiff.ac.uk 


\section{Introduction}

Derivative spectroscopy is conventionally understood to be a collection of techniques for extracting fine structure from spectroscopic data by means of numerical differentiation. An informative account of the history, techniques and scope of the subject may be found in a compendium of seven articles published in O'Haver et al [1]. A more recent discussion about the earlier and current applications of derivative spectroscopy can be found in Anderssen and Hegland [2]. Their paper contains, in Section 2, a review of the early importance of the differentiation of data in terms of the key observation of Lord Rutherford, a summary of some of the novel applications of the methodology, as well as a discussion about the popularity of fourth derivative spectroscopy [3]. In this paper we exploit the power of derivative spectroscopy to achieve peak sharpening and resolution enhancement of spectra.

We shall be exclusively concerned with the recovery of the continuous relaxation spectrum (CRS), $H(\tau)$, from measurements of the storage and loss moduli, $G^{\prime}(\omega)$ and $G^{\prime \prime}(\omega)$. Previous approximations to the CRS have included contributions by Malkin [4], Stadler and Bailly [5], Stadler [6], Davies and Goulding [7], Cho [8] and Anderssen et al [9]. References [5], [6] and [8] are based on cubic spline and polynomial approximations, while references [7] and [9] base their approximations on exact inversion formulae. Wavelets were used in [7] and derivative-based approximations were used in [9]. In our current paper we shall bring together the advantageous properties of both wavelets and derivatives to provide explicit and computable formulae based on exact inversion. Our method facilitates local recovery of the CRS, which is appropriate for real scenarios where the oscillatory shear data are only available for a finite range of frequencies.

Recovery of the CRS involves the solution of one or both of the following Fredholm integral equations of the first kind:

$$
\begin{aligned}
& G^{\prime}(\omega)=G_{e}+\int_{0}^{\infty} \frac{\omega^{2} \tau^{2}}{1+\omega^{2} \tau^{2}} H(\tau) \frac{d \tau}{\tau}, \\
& G^{\prime \prime}(\omega)=\int_{0}^{\infty} \frac{\omega \tau}{1+\omega^{2} \tau^{2}} H(\tau) \frac{d \tau}{\tau} .
\end{aligned}
$$

Our starting point is the Fourier convolution form of equations (1.1) and (1.2):

$$
\begin{aligned}
G^{\prime}(\omega) & =G_{e}+\frac{1}{2}[1+\tanh (\ln \omega)] * H\left(\omega^{-1}\right), \\
G^{\prime \prime}(\omega) & =\frac{1}{2} \operatorname{sech}(\ln \omega) * H\left(\omega^{-1}\right),
\end{aligned}
$$

where convolution is with respect to the logarithmic variable and is defined by

$$
f(\ln \omega) * K\left(\omega^{-1}\right)=f(x) * k(x)=\int_{-\infty}^{\infty} f(x-y) k(y) d y,
$$


where $k(x)=K\left(e^{-x}\right)$ and $x=\ln \omega$. Here it is assumed that $f$ and $K$ are any pair of functions for which the convolution integral exists. Without loss of generality, we shall assume $G_{e}=0$ throughout the paper.

The kernel in (1.4) is of the form $\operatorname{sech}^{\lambda}(\mathrm{x}), \lambda>0$. In the theory of wavelets such kernels are known as scaling functions [see (2.5) below], which act as lowpass filters. The kernel in (1.3) can also be reduced to a scaling function by differentiation. Let $\mathrm{D}$ denote the differential operator

$$
D=\frac{d}{d \ln \omega}=\omega \frac{d}{d \omega},
$$

then

$$
D G^{\prime}(\omega)=\frac{1}{2} \operatorname{sech}^{2}(\ln \omega) * \mathrm{H}\left(\omega^{-1}\right) .
$$

It can also be shown that

$$
\begin{aligned}
G^{\prime \prime}(\omega)-D^{2} G^{\prime \prime}(\omega) & =\operatorname{sech}^{3}(\ln \omega) * \mathrm{H}\left(\omega^{-1}\right), \\
D G^{\prime}(\omega)-\frac{1}{4} D^{3} G^{\prime}(\omega) & =\frac{3}{4} \operatorname{sech}^{4}(\ln \omega) * \mathrm{H}\left(\omega^{-1}\right) .
\end{aligned}
$$

The sech kernels in equations (1.4)-(1.7) are the key to the application of derivative spectroscopy to the recovery of the CRS. Increasing integer powers of the sech function, suitably normalized, form a delta sequence; i.e.

$$
\delta(x)=\lim _{n \rightarrow \infty} \mu_{n}^{-1} \operatorname{sech}^{n}(x), \quad \text { where } \mu_{n}=\int_{-\infty}^{\infty} \operatorname{sech}^{n}(x) d x .
$$

Hence, equations (1.4)-(1.7) give rise to the following sequence of approximations which, when working with exact data, increase in accuracy as the order of the highest derivative increases:

$$
\begin{aligned}
& H\left(\omega^{-1}\right) \approx \frac{2}{\pi} G^{\prime \prime}(\omega), \\
& H\left(\omega^{-1}\right) \approx D G^{\prime}(\omega), \\
& H\left(\omega^{-1}\right) \approx \frac{2}{\pi}\left[G^{\prime \prime}(\omega)-D^{2} G^{\prime \prime}(\omega)\right], \\
& H\left(\omega^{-1}\right) \approx D G^{\prime}(\omega)-\frac{1}{4} D^{3} G^{\prime}(\omega) .
\end{aligned}
$$

The approximation (1.9) is usually attributed to Fuoss and Kirkwood [10]. The approximations (1.10)-(1.12) were first derived by Schwarzl and Staverman [11], but they did not pursue approximations of order greater than three. Tschoegl [12], Friedrich [13] and Anderssen et al [9] have studied the $n$-th order formulae in this sequence. Anderssen et al demonstrated that the Schwarzl-Staverman sequence converges very slowly. It is not difficult to show that, working with exact data, derivatives of order higher than 30 may be required to recover a peak in the CRS to $99 \%$ of its true height. From (1.8), it may be deduced 
that the approximation to $H\left(\omega^{-1}\right)$ obtained from the $n$-th order formula in this sequence is

$$
H\left(\omega^{-1}\right) \approx \mu_{n+1}^{-1} \operatorname{sech}^{n+1}(\ln \omega) * \mathrm{H}\left(\omega^{-1}\right)
$$

For example, taking the unimodal test spectrum $H(\tau)=\frac{2 \tau^{2}}{1+\tau^{4}}$ studied in [9], which has unit height, requires a value of $n \approx 100$ in the Schwarzl-Staverman sequence to recover a height of 0.99 .

In this paper, we use a different derivative-based delta sequence which converges more rapidly than the Schwarzl-Staverman sequence, requiring derivatives of order 10 or less to recover a peak in the CRS to $99 \%$ of its true height. Differentiation is an ill-posed process, the degree of ill-posedness increasing with the order of differentiation. High-order differentiation of experimental data is therefore not normally achievable due to excessive noise amplification. (See, however, the conclusions in [2]). The degree of ill-posedness in $n$-th order differentiation is of algebraic order $n$, (see, for example, Davies and Anderssen [14]), but this is not as severe as in the deconvolution problems (1.3) and (1.4), which are exponentially ill-posed. Davies and Goulding [7] have shown that wavelet regularization can be an effective method for solving (1.3) and (1.4) with experimentally measured data. We shall demonstrate that appropriately constructed wavelet smoothing also makes possible the high-order differentiation of oscillatory shear data.

Derivatives and wavelets are intimately connected, and most of the ideas developed in this paper emerge from this connection. In Section 2, we review the method of wavelet regularization proposed by Davies and Goulding [7], and establish the connection with derivative spectroscopy and delta sequences in Section 3. In Section 4, we obtain formal series expansions for $G^{\prime}(\omega)$ and $G^{\prime \prime}(\omega)$ in terms of weighted Gegenbauer wavelets, while in Section 5 we derive computable inversion formulae for the recovery of the CRS by exploiting the recursive properties of Gegenbauer polynomials. We validate the use of these inversion formulae in Section 6 using both exact and noisy test data. In Section 7 , we invert the polybutadiene blend data used by Honerkamp and Weese [15], and compare the results with previously published results on these data. Conclusions are drawn in Section 8 .

\section{Wavelet regularization}

Throughout this section and the next we shall use the symbol $x$ exclusively to denote $\ln \omega$, and the hat symbol to denote the Fourier transform:

$$
\widehat{f}(p)=\int_{-\infty}^{\infty} f(x) e^{-i p x} d x .
$$

We begin with a brief description of the properties of continuous wavelets. 
A wavelet is a function in the shape of a small wave with zero area. We shall be concerned with real-valued wavelets $\psi \in L^{2}(\mathbb{R})$ defined by the following properties:

(i) $\int_{-\infty}^{\infty} \psi(x) d x=0, \quad$ (the graph of $\psi$ has zero area);

(ii) there exists a constant $C_{\psi}$ such that $0<C_{\psi}=\int_{0}^{\infty} \frac{|\widehat{\psi}(p)|^{2}}{p} d p<\infty$.

The two properties (2.1) and (2.2) are invariant with respect to dilation and translation. Suppose $\sigma>0$ is a fixed scaling parameter and suppose $x_{0}=\ln \omega_{0}$, where $\omega_{0}$ is a fixed reference frequency. Then if $\psi(x)$ is a wavelet, so is $\psi\left(\sigma^{-1}\left(x-x_{0}\right)\right)$.

According to a theorem of Calderón [16], if $H(\tau)$ is any function satisfying

$$
\int_{-\infty}^{\infty} H^{2}(\tau) d \ln \tau<\infty
$$

then $H\left(\omega^{-1}\right)$ may be decomposed into wavelets as follows:

$$
H\left(\omega^{-1}\right)=\frac{1}{C_{\psi}} \int_{0}^{\infty} \psi\left(s^{-1} x\right) * H\left(\omega^{-1}\right) \frac{d s}{s^{2}} .
$$

Here, the wavelet scales, $\mathrm{s}$, range from 0 to $\infty$. For a fixed scale $\sigma>0$, Mallat [17] observes that scales $s>\sigma$ can be collected together into a single term $\phi_{\sigma}(x)$ to give the Calderón-Mallat decomposition

$$
H\left(\omega^{-1}\right)=\frac{1}{C_{\psi}} \phi_{\sigma}(x) * H\left(\omega^{-1}\right)+\frac{1}{C_{\psi}} \int_{0}^{\sigma} \psi\left(s^{-1} x\right) * H\left(\omega^{-1}\right) \frac{d s}{s^{2}},
$$

where

$$
\phi_{\sigma}(x)=\int_{\sigma}^{\infty} \psi\left(s^{-1} x\right) \frac{d s}{s^{2}} .
$$

Equation (2.5) stands as a formal definition of a scaling function since

$$
\phi_{\sigma}(x)=\sigma^{-1} \phi_{1}\left(\sigma^{-1} x\right) .
$$

Davies and Goulding show that if $\phi_{1}$ is chosen as $\phi_{1}(x)=\operatorname{sech}(x)$ then, by applying (2.4), the CRS may be expressed as

$$
H\left(\omega^{-1}\right)=\frac{1}{\pi \sigma} \operatorname{sech}\left(\sigma^{-1} x\right) * H\left(\omega^{-1}\right)+\frac{1}{\pi} \int_{0}^{\sigma} \psi^{*}\left(s^{-1} x\right) * H\left(\omega^{-1}\right) \frac{d s}{s^{2}},
$$

where the wavelet $\psi^{*}$ is given by

$$
\psi^{*}(x)=\operatorname{sech}(x)[1-x \tanh (x)] .
$$


In particular, choosing $\sigma=1$ and bearing in mind that $x=\ln \omega$, it follows from (1.4) that

$$
H\left(\omega^{-1}\right)=\frac{2}{\pi} G^{\prime \prime}(\omega)+\frac{1}{\pi} \int_{0}^{1} \psi^{*}\left(s^{-1} x\right) * H\left(\omega^{-1}\right) \frac{d s}{s^{2}} .
$$

Hence the integral term in (2.8) provides an exact wavelet correction for the Fuoss-Kirkwood approximation (1.9). form

Equation (2.6) is equivalent to a decomposition of the Dirac delta in the

$$
\delta(x)=\frac{1}{\pi \sigma} \operatorname{sech}\left(\sigma^{-1} x\right)+\frac{1}{\pi} \int_{0}^{\sigma} \psi^{*}\left(s^{-1} x\right) \frac{d s}{s^{2}} .
$$

An alternative delta sequence to that of (1.8) is therefore given by

$$
\delta(x)=\frac{1}{\pi \sigma} \operatorname{sech}\left(\sigma^{-1} x\right)+\frac{1}{\pi} \lim _{n \rightarrow \infty} \int_{n^{-1} \sigma}^{\sigma} \psi^{*}\left(s^{-1} x\right) \frac{d s}{s^{2}} .
$$

Since the inversion of equation (1.4) represents a severely ill-posed problem, its solution must be regularized to stabilize the amplification of noise in measured values of $G^{\prime \prime}(\omega)$. Davies and Goulding propose as a regularization the removal of small scales in (2.6). For a fixed value of $\sigma$ in the range $0<\sigma<1$, they choose the regularization $H \rightarrow H_{\sigma}$, where

$$
\begin{aligned}
H_{\sigma}\left(\omega^{-1}\right)= & \frac{1}{\pi \sigma} \operatorname{sech}\left(\sigma^{-1} x\right) * H\left(\omega^{-1}\right)+\frac{1}{\pi \sigma} \psi^{*}\left(\sigma^{-1} x\right) * H\left(\omega^{-1}\right) \\
& =\frac{1}{\pi \sigma} \operatorname{sech}\left(\sigma^{-1} x\right)\left[2-\left(\sigma^{-1} x\right) \tanh \left(\sigma^{-1} x\right)\right] * H\left(\omega^{-1}\right) .
\end{aligned}
$$

It may be shown that the regularized approximation (2.10) may be represented in discrete form by

$$
H_{\sigma}\left(\omega^{-1}\right) \approx \sum_{k=1}^{m} a_{k} \operatorname{sech}\left(\sigma^{-1} \ln \left(\frac{\omega}{\omega_{\mathrm{k}}}\right)\right)
$$

where the unknown coefficients $a_{k}$ can take on both positive and negative values to reflect the change of sign in the kernel function in (2.10). The nodes $\omega_{k}$ are also unknown. The models

$$
\begin{aligned}
G^{\prime}(\omega) & \approx \frac{1}{2} \sum_{k=1}^{m} a_{k}[1+\tanh (\ln \omega)] * \operatorname{sech}\left(\sigma^{-1} \ln \left(\frac{\omega}{\omega_{\mathrm{k}}}\right)\right), \\
G^{\prime \prime}(\omega) & \approx \frac{1}{2} \sum_{k=1}^{m} a_{k} \operatorname{sech}(\ln \omega) * \operatorname{sech}\left(\sigma^{-1} \ln \left(\frac{\omega}{\omega_{\mathrm{k}}}\right)\right)
\end{aligned}
$$

are then fitted to the experimental data by least-squares to determine the coefficients $a_{k}$ and the nodes $\omega_{k}$. 
The parameters $\sigma$ and $m$ in (2.11) act as regularization parameters, and their optimal values can be found by simple searches. The main computational challenge is the determination of the nodes $\omega_{k}$. We shall see below that derivative spectroscopy can provide effective alternatives to the models (2.11)-(2.13) which avoids entirely the determination of nodal distributions.

\section{Derivatives and delta sequences}

In this section, we examine the relationship between the wavelets introduced in the previous section and derivatives of the sech function. This leads to alternative delta sequences to (2.9) which lay the foundation for the derivative spectroscopy of the CRS and its efficient computational implementation in later sections.

Consider the identity

$$
1=\cosh \left(\frac{\pi}{2} \sigma p\right) \operatorname{sech}\left(\frac{\pi}{2} \sigma p\right)
$$

The cosh function is an entire function which admits a Maclaurin series which is everywhere convergent. Replacing the cosh term by its Maclaurin expansion gives

$$
1=\operatorname{sech}\left(\frac{\pi}{2} \sigma p\right)+\sum_{r=1}^{\infty}\left(\frac{\pi}{2}\right)^{2 r} \frac{\sigma^{2 r}}{(2 r) !} p^{2 r} \operatorname{sech}\left(\frac{\pi}{2} \sigma p\right) .
$$

Since the inverse Fourier transform of $\pi \sigma \operatorname{sech}\left(\frac{\pi}{2} \sigma p\right)$ is $\operatorname{sech}\left(\sigma^{-1} x\right)$ we obtain a limit representation of the Dirac delta in the form

$$
\delta(x)=\frac{1}{\pi \sigma} \operatorname{sech}\left(\sigma^{-1} x\right)+\lim _{n \rightarrow \infty} \frac{1}{\pi} \sum_{r=1}^{n}(-1)^{r}\left(\frac{\pi}{2}\right)^{2 r} \frac{\sigma^{2 r-1}}{(2 r) !} D^{2 r} \operatorname{sech}\left(\sigma^{-1} x\right) .
$$

The derivatives $D^{2 r} \operatorname{sech}\left(\sigma^{-1} x\right)$ are all wavelets, and a comparison of (2.9) and (3.1) establishes the link between them and the wavelets $\psi^{*}\left(s^{-1} x\right)$ in the previous section. Indeed, Calderón's theorem tells us that each can be expressed in terms of the other. For example, the similarity between $-D^{2} \operatorname{sech}(x)$ and $\psi^{*}\left(\frac{4}{3} x\right)$ is shown in Figure 1 where plots of the two functions are superposed. This simple example also serves to illustrate the role of scaling.

Consider the delta sequence obtained by truncating the series in (3.1) to $n+1$ terms. This involves even derivatives from order 0 to to order $2 n$. It is informative to compare this sequence with the terms in (1.8) for the same even orders of derivative. In Figure 2 we compare the first four approximations to the $\delta$-function given by the Maclaurin sequence (3.1) with the corresponding evenorder approximations in the Schwarzl-Staverman sequence, i.e. $n=0,2,4,6$ in (1.8). We take $\sigma=1$ in (3.1) so that the zeroth order approximations are the same in both cases. For ease of comparison, the Schwarzl-Staverman sequence is centred at $x=-5$ and shown in blue, while the Maclaurin sequence is centred at $x=5$ and shown in red. The approximations in the former are 
always positive, while those in the latter develop small end-oscillations as $n$ increases. These end-oscillations are a common feature in high-order derivative spectroscopy and play a key role in increasing the resolving power of the sequence. Sequences which are constrained to be positive have considerably less resolving power. This phenomenon is explained by a theorem of Wigner in the context of quantum mechanics. (See Mallat [17], page 142-145). We shall treat the matter of end-oscillations in Section 7 .

In similar fashion to the above, starting from the identity

$$
1=\left(\frac{\pi}{2} \sigma p\right)^{-1} \sinh \left(\frac{\pi}{2} \sigma p\right)\left(\frac{\pi}{2} \sigma p\right) \operatorname{cosech}\left(\frac{\pi}{2} \sigma p\right),
$$

the following limit representation is obtained

$$
\delta(x)=\frac{1}{2 \sigma} \operatorname{sech}^{2}\left(\sigma^{-1} x\right)+\lim _{n \rightarrow \infty} \frac{1}{2} \sum_{r=1}^{n}(-1)^{r}\left(\frac{\pi}{2}\right)^{2 r} \frac{\sigma^{2 r-1}}{(2 r+1) !} D^{2 r} \operatorname{sech}^{2}\left(\sigma^{-1} x\right) .
$$

This representation approaches the limit more rapidly than its partner in (3.1). The first four approximations in the delta sequences obtained from (3.1) and (3.2) are shown in Figure 3, the former on the left in red and the latter on the right in black, both with $\sigma=1$.

Taking the convolutions of both (3.1) and (3.2) with $H\left(\omega^{-1}\right)$, keeping $\sigma=1$, we obtain the following exact inversion formulae for equations (1.4) and (1.5) in terms of the derivatives of $G^{\prime \prime}(\omega)$ and $G^{\prime}(\omega)$ :

$$
\begin{aligned}
& H\left(\omega^{-1}\right)=\frac{2}{\pi} G^{\prime \prime}(\omega)+\frac{2}{\pi} \sum_{r=1}^{\infty}(-1)^{r}\left(\frac{\pi}{2}\right)^{2 r} \frac{1}{(2 r) !} D^{2 r} G^{\prime \prime}(\omega), \\
& H\left(\omega^{-1}\right)=D G^{\prime}(\omega)+\sum_{r=1}^{\infty}(-1)^{r}\left(\frac{\pi}{2}\right)^{2 r} \frac{1}{(2 r+1) !} D^{2 r+1} G^{\prime}(\omega) .
\end{aligned}
$$

These two inversion formulae were derived in a slightly more formal manner in the paper by Anderssen et al [9]. In that paper they were not implemented explicitly, but formed the basis for two derivative based algorithms which utilized Gureyev iteration. This approach replaced the calculation of high-order derivatives with a sequence of convolutions involving second-order derivatives only. This meant that the CRS could be calculated numerically, but not obtained explicity in analytic form. In the next two sections we use (3.3) and (3.4) to derive explicit and computable formulae for the CRS based on weighted Gegenbauer wavelets.

\section{Weighted Gegenbauer wavelets}

Consider the function $\operatorname{sech}^{\lambda-\frac{1}{2}}(x)$, where $\lambda-\frac{1}{2}$ is a positive integer. It is a simple exercise to show that the even order derivatives take the form

$$
D^{2 r} \operatorname{sech}^{\lambda-\frac{1}{2}}(x)=\operatorname{sech}^{\lambda-\frac{1}{2}}(x) P_{r}\left(\tanh ^{2}(x)\right)=\left(1-t^{2}\right)^{\frac{1}{2}\left(\lambda-\frac{1}{2}\right)} P_{r}\left(t^{2}\right),
$$


where $P_{r}\left(t^{2}\right)$ is a polynomial of degree $r$ in $t^{2}$, and $t=\tanh (x),-1 \leq t \leq 1$. For $r \geq 1, D^{2 r} \operatorname{sech}^{\lambda-\frac{1}{2}}(x)$ is a wavelet in $x$, while $\operatorname{sech}^{\lambda-\frac{1}{2}}(x)$ is a scaling function.

Details concerning the Gegenbauer polynomials (ultraspherical polynomials), $C_{n}^{(\lambda)}(t)$, of order $\lambda$ and degree $n$, may be found in [20]. They are orthogonal on the interval $[-1,1]$, with respect to the weight function $\left(1-t^{2}\right)^{\lambda-\frac{1}{2}}$, i.e.

$$
\int_{-1}^{1}\left(1-t^{2}\right)^{\lambda-\frac{1}{2}} C_{m}^{(\lambda)}(t) C_{n}^{(\lambda)}(t) d t=0, \quad m \neq n
$$

The normalization constant is

$$
\mu_{n}^{(\lambda)}=\int_{-1}^{1}\left(1-t^{2}\right)^{\lambda-\frac{1}{2}}\left[C_{n}^{(\lambda)}(t)\right]^{2} d t=2^{1-2 \lambda} \pi \frac{\Gamma(n+2 \lambda)}{(n+\lambda) \Gamma^{2}(\lambda) \Gamma(n+1)} .
$$

Let us define

$$
\psi_{n}^{(\lambda)}(x)=\operatorname{sech}^{\lambda-\frac{1}{2}}(x) C_{n}^{(\lambda)}(\tanh (x)) .
$$

In terms of $t$ we may write

$$
\psi_{n}^{(\lambda)}(x)=\phi_{n}^{(\lambda)}(t)=\left(1-t^{2}\right)^{\frac{1}{2}\left(\lambda-\frac{1}{2}\right)} C_{n}^{(\lambda)}(t) .
$$

It follows from (4.2) that

$$
\int_{-1}^{1} \phi_{m}^{(\lambda)}(t) \phi_{n}^{(\lambda)}(t) d t=0, \quad m \neq n
$$

The set $\left\{\phi_{n}^{(\lambda)}(t)\right\}_{n=0}^{\infty}$ is a complete orthogonal system over [-1,1] with unit weight.

The values of $\lambda$ which best suit our purpose are the half-integer values $\lambda=$ $\frac{3}{2}, \frac{5}{2}, \frac{7}{2}, \ldots$. This means that $2 \lambda+1$ is an even integer. Throughout the rest of this paper we shall assume

$$
2 \lambda+1 \text { is an even integer } \geq 4 .
$$

Under this assumption we shall demonstrate that the function $\operatorname{sech}^{\lambda+\frac{3}{2}}(x) \phi_{n}^{(\lambda)}(\tanh (x))$ is a wavelet in the variable $x$ when $n \geq 1$. We shall write this wavelet in the form

$$
\gamma_{n}^{(\lambda)}(x)=\operatorname{sech}^{2 \lambda+1}(x) C_{n}^{(\lambda)}(\tanh (x)), \quad n \geq 1 .
$$

Changing the variable in (4.2) from $t$ to $x$ and choosing $m=0$ gives

$$
\int_{-\infty}^{\infty} \gamma_{n}^{(\lambda)}(x) d x=0, \quad n \geq 1
$$


since $C_{0}^{(\lambda)}(t)=1$. Furthermore, under the assumption $(4.7), \gamma_{n}^{(\lambda)}(x)$ can be expressed as the derivative of a polynomial of degree $n+2 \lambda$ in $\tanh (\mathrm{x})$, i.e.

$$
\gamma_{n}^{(\lambda)}(x)=D P_{n+2 \lambda}(\tanh (x)), \quad n \geq 1
$$

[See (4.23) below]. Equations (4.9) and (4.10), together with the fact that $\gamma_{n}^{(\lambda)}(x) \in L^{2}(\mathbb{R})$, are sufficient to prove that $\gamma_{n}^{(\lambda)}(x)$, as defined by $(4.8)$, is a wavelet. [See equations (2.1) and (2.2)].

The maximum value of $\left|\gamma_{n}^{(\lambda)}(x)\right|$ grows very slowly with $n$. With $\lambda=\frac{3}{2}$, the two wavelets $\gamma_{4}^{(\lambda)}(x)$ and $\gamma_{16}^{(\lambda)}(x)$ are shown in Figure 4 .

We shall refer to the wavelet in (4.8) as a weighted Gegenbauer wavelet. We have found no references to the existence of this wavelet in the literature. However, there are many references to other types of wavelet based on Gegenbauer polynomials (see, for example, [18] and [19].)

We shall now construct a wavelet expansion for $G^{\prime \prime}(\omega)$ in the form

$$
G_{N}^{\prime \prime}(\omega)=\sum_{n=0}^{N} a_{n}^{(\lambda, N)} \gamma_{n}^{(\lambda)}(x), \quad x=\ln \omega
$$

which, as $N \rightarrow \infty$, converges to $G^{\prime \prime}(\omega)$ in a weighted $L^{2}$-norm, i.e.

$$
\lim _{N \rightarrow \infty} \int_{-\infty}^{\infty} \operatorname{sech}^{2}(x)\left|G^{\prime \prime}(\omega)-\sum_{n=0}^{N} a_{n}^{(\lambda, N)} \gamma_{n}^{(\lambda)}(x)\right|^{2} d x=0
$$

The series (4.11) has the mathematical structure

$$
G^{\prime \prime}(\omega)=\text { scaling function + wavelet series, }
$$

a structure which is shared with (3.1) and (3.2). For ease of reference, we shall refer to the expansion in (4.11) as a wavelet expansion, notwithstanding the presence of the initial scaling function.

To construct (4.11) we first expand $G^{\prime \prime}(\omega)$ as an orthogonal series in the variable $t=\tanh (x)$ on the interval $[-1,1]$. Let $G^{\prime \prime}(\omega)=\zeta(t)$ and let $\nu$ be the half-integer such that $\nu-\frac{1}{2}=2 \lambda+1$. We may then write

$$
\zeta(t)=\sum_{n=0}^{\infty} a_{n}^{(\nu)} \phi_{n}^{(\nu)}(t), \quad-1 \leq t \leq 1,
$$

where

$$
\begin{aligned}
a_{n}^{(\nu)} & =\left[\mu_{n}^{(\nu)}\right]^{-1} \int_{-1}^{1} \zeta(t) \phi_{n}^{(\nu)}(t) d t \\
& =\left[\mu_{n}^{(\nu)}\right]^{-1} \int_{-\infty}^{\infty} \operatorname{sech}^{2}(x) G^{\prime \prime}(\omega) \psi_{n}^{(\nu)}(x) d x
\end{aligned}
$$


Let us truncate the series in (4.13) and write

$$
G_{N}^{\prime \prime}(\omega)=\sum_{n=0}^{N} a_{n}^{(\nu)} \phi_{n}^{(\nu)}(t)
$$

We can express this in the form

$$
G_{N}^{\prime \prime}(\omega)=\sum_{n=0}^{N} a_{n}^{(\nu)} \operatorname{sech}^{\nu-\frac{1}{2}}(x) C_{n}^{(\nu)}(\tanh (x)),
$$

and expand the polynomial $C_{n}^{(\nu)}(t)$ in terms of the basis $\left\{C_{m}^{(\lambda)}(t)\right\}_{m=0}^{n}$, i.e. we can write

$$
\begin{aligned}
C_{n}^{(\nu)}(t) & =\sum_{m=0}^{n} \rho_{m n} C_{m}^{(\lambda)}(t) \\
\rho_{m n} & =\left[\mu_{m}^{(\lambda)}\right]^{-1} \int_{-1}^{1}\left(1-t^{2}\right)^{\lambda-\frac{1}{2}} C_{n}^{(\nu)}(t) C_{m}^{(\lambda)}(t) .
\end{aligned}
$$

Hence (4.16) takes the form

$$
G_{N}^{\prime \prime}(\omega)=\sum_{m=0}^{N} a_{m}^{(\lambda, N)} \operatorname{sech}^{2 \lambda+1}(x) C_{m}^{(\lambda)}(\tanh (x)),
$$

where

$$
a_{m}^{(\lambda, N)}=\sum_{n=0}^{N} \rho_{m n} a_{n}^{(\nu)}
$$

The equation (4.18) is the same as (4.11), and so our construction is complete.

Note that the coefficients in (4.11) depend on $N$, whereas the coefficients in (4.15) are independent of $N$. This is because the wavelet basis in (4.11) is not orthogonal, whereas the basis in (4.15) is orthogonal. The convergence property (4.12) follows immediately from the $L^{2}$-convergence of the orthogonal series (4.13):

$$
\lim _{N \rightarrow \infty} \int_{-1}^{1}\left|\zeta(t)-\sum_{n=0}^{N} a_{n}^{(\nu)} \phi_{n}^{(\nu)}(t)\right|^{2} d t=0
$$

In exactly similar fashion we can derive a wavelet expansion for $D G^{\prime}(\omega)$. The series

$$
D G_{N}^{\prime}(\omega)=\sum_{n=0}^{N} b_{n}^{(\nu)} \phi_{n}^{(\nu)}(t)
$$

may be written in the form

$$
D G_{N}^{\prime}(\omega)=\sum_{n=0}^{N} b_{n}^{(\lambda, N)} \gamma_{n}^{(\lambda)}(x)
$$


and this expansion too converges to $D G^{\prime}(\omega)$ in the same weighted- $L^{2}$-norm as in (4.12). The corresponding expansion for $G^{\prime}(\omega)$, however, is not a wavelet expansion. Under the constraint (4.7) it can easily be shown that

$$
G_{N}^{\prime}(\omega)=\sum_{n=0}^{N} b_{n}^{(\lambda, N)} P_{n+2 \lambda}(\tanh (x))
$$

where $P$ is the polynomial of degree $n+2 \lambda$ defined by

$$
P_{n+2 \lambda}(t)=\int_{-1}^{t}\left(1-s^{2}\right)^{\lambda-\frac{1}{2}} C_{n}^{(\lambda)}(s) d s .
$$

We have mentioned in $\S 2$ that wavelets remain wavelets under dilation and translation of the variable $x$. We shall need to exploit this property if we are to implement effective wavelet smoothing of experimental oscillatory shear data. To this end, we redefine the variable $x=\ln \omega$ as

$$
x=\sigma^{-1} \ln \left(\frac{\omega}{\omega_{0}}\right)
$$

where $\sigma>0$, and $\omega_{0}$ is a reference frequency. The definition (4.24) will remain in force henceforth.

To summarize, the purpose of this section was to derive convergent series for $G^{\prime \prime}(\omega)$ and $D G^{\prime}(\omega)$ in terms of weighted Gegenbauer wavelets. In the next section we obtain series for the CRS by accessing the special properties of the Gegenbauer wavelets.

\section{Wavelet expansions for the continuous relaxation spectrum}

The $n$th degree Gegenbauer polynomial of order $\lambda$ may be defined by the Rodrigues formula [see 20]

$$
C_{n}^{(\lambda)}(t)=\frac{(-2)^{n}}{n !} \frac{\Gamma(n+\lambda) \Gamma(n+2 \lambda)}{\Gamma(\lambda) \Gamma(2 n+2 \lambda)}\left(1-t^{2}\right)^{-\lambda+\frac{1}{2}} \frac{d^{n}}{d t^{n}}\left[\left(1-t^{2}\right)^{n+\lambda-\frac{1}{2}}\right] .
$$

Of the many relations shared by these polynomials we shall require the following two identities:

$$
\begin{aligned}
\left(1-t^{2}\right) \frac{d}{d t} C_{n}^{(\lambda)}(t) & =(n+2 \lambda) t C_{n}^{(\lambda)}(t)-(n+1) C_{n+1}^{(\lambda)}(t), \\
2(n+\lambda) t C_{n}^{(\lambda)}(t) & =(n+1) C_{n+1}^{(\lambda)}(t)+(n+2 \lambda-1) C_{n-1}^{(\lambda)}(t) .
\end{aligned}
$$

In consequence of (4.24) the operator $D$ can be written

$$
D=\frac{d}{d \ln \omega}=\sigma^{-1}\left(1-t^{2}\right) \frac{d}{d t}, \quad t=\tanh \left[\sigma^{-1} \ln \left(\frac{\omega}{\omega_{0}}\right)\right]
$$


Hence, from (5.2) and (5.3) we can deduce

$$
D \gamma_{n}^{(\lambda)}(x)=\sigma^{-1}\left[A_{n-1} \gamma_{n-1}^{(\lambda)}(x)+B_{n+1} \gamma_{n+1}^{(\lambda)}(x)\right],
$$

where $A_{n}$ and $B_{n}$ are constants defined by

$$
A_{n}=\frac{n(n+2 \lambda)}{2(n+\lambda+1)}, \quad \text { and } \quad B_{n}=-\frac{n(n+2 \lambda)}{2(n+\lambda-1)} .
$$

Differentiating once more, we find

$$
D^{2} \gamma_{n}^{(\lambda)}(x)=\sigma^{-2}\left[\chi_{n,-1}^{[1]} \gamma_{n-2}^{(\lambda)}(x)+\chi_{n 0}^{[1]} \gamma_{n}^{(\lambda)}(x)+\chi_{n 1}^{[1]} \gamma_{n+2}^{(\lambda)}(x)\right],
$$

where

$$
\chi_{n,-1}^{[1]}=A_{n-1} A_{n-2}, \quad \chi_{n 0}^{[1]}=A_{n-1} B_{n}+A_{n} B_{n+1}, \quad \text { and } \chi_{n 1}^{[1]}=B_{n+1} B_{n+2} .
$$

For $r \geq 1$, the corresponding expression for $D^{2 r} \gamma_{n}^{(\lambda)}(x)$ is

$$
D^{2 r} \gamma_{n}^{(\lambda)}(x)=\sigma^{-2 r} \sum_{k=-r}^{r} \chi_{n k}^{[r]} \gamma_{n+2 k}^{(\lambda)}(x)
$$

where the constants $\chi_{n k}^{[r]}$ are computed from the recurrences

$$
\chi_{n k}^{[r+1]}=\chi_{n, k+1}^{[r]} \chi_{n+2 k+2,-1}^{[1]}+\chi_{n k}^{[r]} \chi_{n+2 k, 0}^{[1]}+\chi_{n, k-1}^{[r]} \chi_{n+2 k-2,1}^{[1]},
$$

for $-(r+1) \leq k \leq r+1$,

with the constraint that

$$
\chi_{n k}^{[r]}=0 \text { if }|k|>r .
$$

The constants $\chi_{n k}^{[r]}$ grow like $n^{2 r}$, which reflects the fact that differentiation of order $2 r$ has an associated degree of ill-posedness equal to $2 r$.

Substituting (5.9) into (4.11) gives

$$
D^{2 r} G_{N}^{\prime \prime}(\omega)=\sigma^{-2 r} \sum_{n=0}^{N} \sum_{k=-r}^{r} \chi_{n k}^{[r]} a_{n}^{(\lambda, N)} \gamma_{n+2 k}^{(\lambda)}(x) .
$$

Finally we may obtain a wavelet expansion for the CRS from (3.3), in the form

$$
H_{N}\left(\omega^{-1}\right)=\frac{2}{\pi} \sum_{n=0}^{N} \sum_{r=0}^{\infty} \sum_{k=-r}^{r} \alpha_{n r k}^{(\lambda, N)} \gamma_{n+2 k}^{(\lambda)}(x),
$$

where the coefficients $\alpha_{n r k}^{(\lambda, N)}$ are given by

$$
\alpha_{n r k}^{(\lambda, N)}=(-1)^{r}\left(\frac{\pi}{2}\right)^{2 r} \frac{\sigma^{-2 r}}{(2 r) !} \chi_{n k}^{[r]} a_{n}^{(\lambda, N)},
$$


and it is understood that $\chi_{n 0}^{[0]}=1$.

For fixed $n$, the coefficients $\alpha_{n r k}^{(\lambda, N)}$ decay at a rate no slower than $[(2 r) !]^{-1}\left(\sigma^{-1} \frac{\pi}{2} N\right)^{2 r}$ as $r$ increases, while, for fixed $r$, they decay like $n^{-2 \lambda}$ as $n$ increases. It can be shown that the coefficients $a_{n}^{(\nu)}$ decay at a rate marginally slower than $n^{-\nu+\frac{1}{2}}$ as $n$ increases. For fixed $N$, therefore, the series (5.13) converges. However, the reader should not be deceived by this seemingly benign attribute. The formula $(5.13)$ is, in effect, a high-order differentiation rule, as is evident from (3.3). It carries in its implementation a noise amplification factor which grows rapidly with $N$. We shall return to this in Section 6 .

A discussion of convergence of the series (5.13) in the limit $N \rightarrow \infty$ is beyond the scope of this paper. In working with exact data it is possible to demonstrate numerical convergence with increasing $N$. On the other hand, in working with experimental data, both $N$ and $r$ must be kept finite. We shall give further details in Sections 6 and 7 .

We may obtain a second wavelet expansion for the CRS from (3.4), in the form

$$
H_{N}\left(\omega^{-1}\right)=\sum_{n=0}^{N} \sum_{r=0}^{\infty} \sum_{k=-r}^{r} \beta_{n r k}^{(\lambda, N)} \gamma_{n+2 k}^{(\lambda)}(x)
$$

where the coefficients $\beta_{n r k}^{(\lambda, N)}$ are given by

$$
\beta_{n r k}^{(\lambda, N)}=(-1)^{r}\left(\frac{\pi}{2}\right)^{2 r} \frac{\sigma^{-(2 r+1)}}{(2 r+1) !} \chi_{n k}^{[r]} b_{n}^{(\lambda, N)} .
$$

This expansion also converges for fixed $N$. Like (5.13), the formula (5.15) is, in effect, a high-order differentiation rule with an associated noise amplification factor.

We have therefore achieved our goal of presenting computable inversion formulae for the CRS in terms of the coefficients in the wavelet expansions for $G^{\prime \prime}(\omega)$ and $D G^{\prime}(\omega)$, where it is not necessary to differentiate the data to obtain the coefficients for $D G^{\prime}(\omega)$. In the next section we shall validate the series (5.13) and (5.15) numerically.

\section{Numerical validation}

We first address the question of how to choose the scaling parameter $\sigma$. In Section 2 , the wavelet $\psi^{*}\left(\sigma^{-1} x\right)$ had a single associated scale $\sigma$, which was chosen in the range $0<\sigma<1$. However, the wavelet $\gamma_{n}^{(\lambda)}(x)$ contains multiple scales ranging from the largest value of $\sigma(2 \lambda+1)^{-1}$ to the smallest value of 
approximately $\sigma(n+2 \lambda+1)^{-1}$. To account exactly for the asymptotic behaviour of $G^{\prime}(\omega)$ and $G^{\prime \prime}(\omega)$ as $\omega \rightarrow 0$ and $\omega \rightarrow \infty$, it is necessary to choose

$$
\sigma(2 \lambda+1)^{-1}=1 .
$$

This means that in the context of Gegenbauer wavelets we must choose $\sigma \geq 4$ to provide exact asymptotics for $G^{\prime}(\omega)$ and $G^{\prime \prime}(\omega)$. However, when smoothing experimental data over a limited range of frequencies, exact asymptotics become marginally less important, and can give way to optimal smoothing and regularization criteria. When working with experimental data, therefore, we relax the constraint (6.1) a little and allow

$$
0.8<\sigma(2 \lambda+1)^{-1}<1.2 .
$$

Once a regularized CRS has been determined, the models for $G^{\prime}(\omega)$ and $G^{\prime \prime}(\omega)$ can be postprocessed using (1.3) and (1.4), and the exact asymptotics restored.

Another important practical consideration is how many derivatives to include in the series (3.3) and (3.4). Our numerical experiments have shown that, for fixed $N$, the series (5.13) converges rapidly with respect to $r$. The series (5.15) converges even faster, as should be evident by comparing the dependence on $r$ of the coefficients in (5.14) and (5.16). Numerical convergence is obtainable in (5.13) with $r \leq 5$, and is obtainable in (5.15) with $r \leq 4$. This means that derivatives of order greater than 10 are not required in (3.3) and order greater than 9 in (3.4). We shall adopt these limits on the highest order of differentiation throughout.

A third question concerns the consistency of the two spectra obtained from (3.3) and (3.4), and implemented via (5.13) and (5.15), respectively. To avoid confusion we refer to the spectrum obtained from the loss modulus alone, i.e. from (5.13), as the loss spectrum and that obtained from the storage modulus alone, from (5.15), as the storage spectrum . In a perfect scenario, working with data than can be differentiated without error, the two spectra would be in perfect agreement. However this can never be the case, since we must work with sampled data which is subject to observational or computational error.

Suppose, first, that we have determined the loss modulus from (5.13). The spectrum can be used to recompute (postprocess) both $G^{\prime}$ and $G^{\prime \prime}$ from (1.3) and (1.4). The postprocessed $G^{\prime \prime}$ should agree with the original loss modulus data used to determine the loss spectrum. The postprocessed $G^{\prime}$ should also be consistent with the storage modulus data which was not used in determining the loss spectrum. The same scenario holds when the storage modulus is obtained from (5.15). The postprocessed $G^{\prime \prime}$ should be consistent with the loss modulus data which was not used in its determination.

The overall consistency of fit to both sets of data is effectively optimized by taking a weighted average of the loss spectrum and the storage spectrum. The 
choice of weights depends on the relative quality of the $G^{\prime}$ and $G^{\prime \prime}$-data, the sampling points, and to some extent the range of frequencies sampled. Let $\xi$ be a number between 0 and 1, and consider a weighted average of the storage and loss spectra in the form

$$
H_{\xi}=(1-\xi) H_{\text {storage }}+\xi H_{\text {loss }} .
$$

Let $\bar{G}_{\xi}^{\prime}$ and $\bar{G}_{\xi}^{\prime \prime}$ denote the storage and loss moduli calculated from $H_{\xi}$, and let $K$ denote the number of sampled frequencies $\omega_{k}$. Following a standard approach, the weights $\xi$ and $1-\xi$ can be chosen to minimize the combined sum of squared residuals

$$
\sum_{k=1}^{K}\left[\left(1-\frac{\bar{G}_{\xi}^{\prime}\left(\omega_{k}\right)}{G^{\prime}\left(\omega_{k}\right)}\right)^{2}+\left(1-\frac{\bar{G}_{\xi}^{\prime \prime}\left(\omega_{k}\right)}{G^{\prime \prime}\left(\omega_{k}\right)}\right)^{2}\right] .
$$

In this section, we look at synthetic data from a known spectrum, namely the double log-normal spectrum of Honerkamp and Weese [15], given by

$$
H(\tau)=\frac{1}{2 \sqrt{(2 \pi)}}\left\{\exp \left[-\frac{1}{2}\left(\ln \tau-\ln \tau_{1}\right)^{2}\right]+\exp \left[-\frac{1}{2}\left(\ln \tau-\ln \tau_{2}\right)^{2}\right]\right\}
$$

where the two peaks are centred at $\tau_{1}=5 * 10^{-2},\left(\ln \tau_{1}=-2.996\right)$ and $\tau_{2}=5$, $\left(\ln \tau_{2}=1.609\right)$, and are of equal height. The spectrum is normalized so that

$$
G^{\prime}(\infty)=\int_{-\infty}^{\infty} H(\tau) d \ln \tau=1
$$

$G^{\prime}(\omega)$ and $G^{\prime \prime}(\omega)$ are calculated by numerical quadrature to 8 significant figures at 30 values of frequency in the range $10^{-2} \leq \omega \leq 10^{3}$ corresponding to equal spaced values of $\ln \omega$ in the range $-6.91<\ln \omega<6.91$, with a sampling interval of 0.476 . We have chosen exactly the same frequency sampling points as reported in McDougall et al [21]. We shall refer to this data set as precision data since the noise level is less than $5 \times 10^{-9}$. We shall quote all frequencies and relaxation times in natural-log values.

The purpose of a reference frequency $\omega_{0}$ is to estimate where the central point of the recovered spectrum should be. If the central point is $\ln \tau_{0}$, then $\omega_{0}$ should be chosen so that $\ln \omega_{0}=-\ln \tau_{0}$. If possible we look for extrema in the $G^{\prime \prime}$-data. If there is only one maximum, this gives an indication of where $\omega_{0}$ should be chosen. Similarly if there is only one minimum. If there are more than two maxima or minima, the situation is less clear, but some central point within the range of the outlying extrema should be chose for $\omega_{0}$. Figure 6 shows the exact spectrum for the double log-normal CRS in black points. The sampled precision data for $G^{\prime}$ is shown in blue points, and those for $G^{\prime \prime}$ in red points. When looking at $G^{\prime \prime}(\omega)$ it is obvious that there is a minimum halfway between the two peaks, i.e. at $\ln \omega=0.6935$. We choose this value for $\ln \omega_{0}$. 
We now collect together the steps needed to apply the derivative spectroscopy approach we have developed in previous sections. We describe two simple algorithms, one for recovering the loss spectrum from the $G^{\prime \prime}$-data, and the other for recovering the storage spectrum from the $G^{\prime}$-data. It is usually necessary to implement the loss spectrum algorithm first.

\section{The loss spectrum algorithm}

Step 1. Choose $N, \sigma, \ln \omega_{0}$ and $\lambda$.

Step 2. Find the $N+1$ coefficients $\left.\left\{a_{n}^{(\lambda, N}\right)\right\}_{n=0}^{N}$ by least-squares fit of the model (4.11) to the $G^{\prime \prime}$-data.

Step 3. Compute the loss spectrum from (5.13) with $r$ in the range $0 \leq r \leq 5$. Step 4. Test the compatibility of the recovered spectrum $H_{N}$ with both sets of data $G^{\prime}$ and $G^{\prime \prime}$, i.e. compute

$$
\begin{aligned}
& \bar{G}_{N}^{\prime}(\omega)=\frac{1}{2}[1+\tanh (\ln \omega)] * \mathrm{H}_{\mathrm{N}}\left(\omega^{-1}\right), \\
& \bar{G}_{N}^{\prime \prime}(\omega)=\frac{1}{2} \operatorname{sech}(\ln \omega) * \mathrm{H}_{\mathrm{N}}\left(\omega^{-1}\right),
\end{aligned}
$$

and obtain some measure of the discrepancies $G^{\prime}(\omega)-\bar{G}_{N}^{\prime}(\omega)$ and $G^{\prime \prime}(\omega)-$ $\bar{G}_{N}^{\prime \prime}(\omega)$.

Step 5. If the discrepancies in Step 4 are below a chosen tolerance level, stop. Otherwise return to Step 1 and repeat Steps $1-5$.

Step 6. Calculate an estimated value for $G^{\prime}(\infty)$ using

$$
G^{\prime}(\infty) \approx \int_{-\infty}^{\infty} H_{N}(\tau) d \ln \tau
$$

\section{The storage spectrum algorithm}

Step 1. Include an extra datum in the $G^{\prime}$-data set, namely the value of $G^{\prime}(\infty)$ calculated at the end of the loss spectrum algorithm. This is required to achieve consistency between the values of $G^{\prime}(\infty)$ obtained from both algorithms.

Step 2. Choose $N, \sigma, \ln \omega_{0}$ and $\lambda$.

Step 3. Find the $N+1$ coefficients $\left.\left\{b_{n}^{(\lambda, N}\right)\right\}_{n=0}^{N}$ by least-squares fit of the model (4.22) to the $G^{\prime}$-data.

Step 4. Compute the storage spectrum from (5.15) with $r$ in the range $0 \leq r \leq 4$.

Step 5. Test the compatibility of the recovered spectrum $H_{N}$ with both sets of data $G^{\prime}$ and $G^{\prime \prime}$, i.e. compute $\bar{G}_{N}^{\prime}(\omega)$ and $\bar{G}_{N}^{\prime \prime}(\omega)$ from $(6.5)$ and (6.6), and obtain a measure of the discrepancies $G^{\prime}(\omega)-\bar{G}_{N}^{\prime}(\omega)$ and $G^{\prime \prime}(\omega)-\bar{G}_{N}^{\prime \prime}(\omega)$.

Step 6. If the discrepancies in Step 5 are below a chosen tolerance level, stop. Otherwise return to Step 2 and repeat Steps $2-6$.

Let $\Omega$ represent the set of sampled frequencies. As measures of the discrepancies between the sampled data and their continuous representation by the models (6.5) and (6.6), we take the two values

$$
\epsilon_{N}^{\prime}=\max _{\omega \in \Omega}\left|G^{\prime}(\omega)-\bar{G}_{N}^{\prime}(\omega)\right| \quad \text { and } \quad \epsilon_{\mathrm{N}}^{\prime \prime}=\max _{\omega \in \Omega}\left|\mathrm{G}^{\prime \prime}(\omega)-\overline{\mathrm{G}}_{\mathrm{N}}^{\prime \prime}(\omega)\right| .
$$


For the precision data, we take as an acceptable tolerance level the value $5 \times 10^{-5}$ which is $0.01 \%$ of the mean value of $G^{\prime}$. Applying the loss spectrum algorithm to the precision $G^{\prime \prime}$-data, we choose $\sigma=4, \ln \omega_{0}=0.6935$, as previously discussed, and $\lambda=\frac{3}{2}$. $\quad N$ is increased until the chosen tolerance level is reached, which occurs when $N=16$ with $\epsilon_{N}^{\prime}=1.5 \times 10^{-5}$ and $\epsilon_{N}^{\prime \prime}=5.4 \times 10^{-6}$. The wavelet expansion which represents $G^{\prime \prime}$ is shown as the red curve in Figure 5, a linear plot of the loss spectrum $H_{16}$ is shown as a continuous black line in Figure 6, and the corresponding curves for $\bar{G}_{16}^{\prime}$ and $\bar{G}_{16}^{\prime \prime}$ are shown in blue and red, respectively, in Figure 6. A log-log plot of the loss spectrum, compared with the exact spectrum, is shown in Figure 7. The maximum error in the recovered loss spectrum is $4.8 \times 10^{-4}$, which is consistent with a noise amplification factor of about 100 . The value of $G^{\prime}(\infty)$ obtained from $H_{16}$ is 0.9994 , compared with the exact value of 1 .

Normally, a noise amplification factor of 100 would not be sustainable. However, it is acceptable when $\epsilon^{\prime}$ and $\epsilon^{\prime \prime}$ are as small as $10^{-5}$ which is the case with the precision data. With the value of $N$ as high as 16 , the recovered spectrum is also highly sensitive to small changes in $\sigma$. When working with the storage spectrum algorithm, a value of $N=16$ again gives acceptable results with $\ln \omega_{0}=0.6935$ and $\lambda=\frac{3}{2}$, even though the noise amplification factor is higher, around 300. To achieve plots which are indistinguishable from those in Figures 6 and 7 we need to choose a value of $\sigma=4.0176$, which gives $\sigma(2 \lambda+1)^{-1}=1.0044$, well within the range of (6.2). The wavelet expansion which represents $G^{\prime}$ is shown as the blue curve in Figure 5. The sensitivity to small changes in $\sigma$ is even greater than previously due to the larger noise amplification factor.

Fortunately, for smaller values of $N$, the noise amplification factor can be as low as $O(1)$. We shall demonstrate this for the noisy data used by McDougall et al [21], where $4 \%$ randomly generated white noise has been added to each datum for the double log-normal spectrum studied above. (We are grateful to these authors for giving us access to their data). The wavelet expansion which represents $G^{\prime \prime}$ is shown as the red curve in Figure 8. Figure 9 shows the results of the loss spectrum algorithm applied to the noisy $G^{\prime \prime}$-data. The chosen values here are $N=6, \sigma=4.44, \ln \omega_{0}=0.6935$, and $\lambda=\frac{3}{2}$. The fit to the noisy data have maximum discrepancies $\epsilon_{N}^{\prime}=6.1 \times 10^{-2}$ and $\epsilon_{N}^{\prime \prime}=7.7 \times 10^{-3}$, with an RMS error of fit of $6 \%$ for $G^{\prime}$ and $4.8 \%$ for $G^{\prime \prime}$, consistent with the noise level in the data. The maximum error in the recovered spectrum is $2.2 \times 10^{-2}$, with a noise amplification factor of less than 3 . Also, the sensitivity of the recovered spectrum to small changes in $\sigma$ is much reduced. Finally, the value of $G^{\prime}(\infty)$ obtained from $H_{6}$ is 0.9989 , compared with the exact value of 1 .

When determining the storage spectrum from the $G^{\prime}$-data, two iterations of the storage algorithm are required. See Figure 8. The choice of parameters $N=5, \sigma=3.8, \ln \omega_{0}=0.55$, and $\lambda=\frac{3}{2}$ leads to the results shown in Figure 10. The maximum discrepancy $\epsilon_{N}^{\prime}$ is the same as the largest error in the storage 
spectrum, both around $6 \times 10^{-2}$. This means there is no noise amplification of significance. The storage spectrum in Figure 10 differs from the loss spectrum in Figure 9, but the fits to the $G^{\prime}$-data and $G^{\prime \prime}$-data obtained from it are virtually unchanged from those determined from the loss spectrum. The value of $G^{\prime}(\infty)$ is 1.0107, compared with the exact value of 1 . A weighted average of the two spectra is shown in Figure 11, with a weight of 0.4 given to the loss spectrum and 0.6 to the storage. The recalculated $\bar{G}_{N}^{\prime}$ and $\bar{G}_{N}^{\prime \prime}$ obtained from this spectrum are shown in Figure 12.

McDougall et al [21] have compared the results of three different methods for determining this CRS from their noisy $G^{\prime}, G^{\prime \prime}$-data. They compared the methods of Cho and Park [22] (fixed point iteration); Honerkamp and Weese [23] (NLREG) and Stadler and Bailly [5] (cubic splines). In the central range of relaxation times, $0.1<\tau<10$, our result in Figure 11 shows greater accuracy than the three methods quoted by McDougall et al. Outside this range our result shows comparable accuracy.

\section{Working with experimental data: the effect of limited sampling.}

The sampled synthetic data in the previous example spanned sufficient frequencies for the CRS to be recovered without any end-effects due to a limited frequency range. In this section we work with a limited range of experimental data. We use the data published by Honerkamp and Weese [15] for a polybutadiene blend, which we refer to as PBD1. This choice of data enables us to compare results generated in this section with published results obtained by other methods.

The experimental data and their wavelet expansion representations are shown in Figure 13. There are 17 sampled frequencies, in the range $2.413 s^{-1}<\omega<1114 s^{-1}$, or $0.91<\ln \omega<7.02$. The $G^{\prime \prime}$-data show a single maximum in the region of $\ln \omega=4$. The loss spectrum, storage spectrum and averaged CRS are shown separately in Figure 14. The parameters in determining the loss spectrum are $N=5, \sigma=3.4, \ln \omega_{0}=3.95$ and $\lambda=\frac{3}{2}$, and in determining the storage spectrum are $N=5, \sigma=3.64, \ln \omega_{0}=4.34$ and $\lambda=\frac{3}{2}$. Both loss and storage spectra show small end-oscillations. The averaged spectrum is therefore truncated at a terminal relaxation time of $\ln \tau=-2.6$. The $G^{\prime}$ - and $G^{\prime \prime}$-models calculated from this spectrum shows excellent reproducibility of the data, as seen from Figure 15. Linear plots are shown in Figure 16.

Previously published results on the PBD1 spectrum may be found in the papers by Honerkamp and Weese [15] using Tikhonov regularization, Davies and Goulding [7] using wavelet regularization, and Anderssen et al [9] using Gureyev iteration. The spectrum in Figure 16, obtained using the algorithms described in Section 6, is in excellent agreement with the spectra found in [7] and [9]. All three spectra are non-negative and bimodal with the larger 
peak positioned at a relaxation time $\tau_{1}=1.7 \times 10^{-2}$ in all three cases. To compare the spectrum of Figure 9 with that obtained by Honerkamp and Weese, the spectrum needs to be normalized. The comparison is made in Figure 17. In the Honerkamp and Weese spectrum the position the larger peak is more difficult to determine but is clearly quite close to the value $\tau_{1}=1.7 \times 10^{-2}$ obtained by the three other methods. However, the nature of the filter associated with Tikhonov regularization makes the Honerkamp and Weese spectrum broader than the spectra determined by the other methods.

The theory of sampling localization (see [21] and [24]) tells us that the larger of the two peaks lies within the reduced reciprocal range of the sampled frequencies. Accuracy, on the other hand, depends on the method of calculation, as is evident from Figure 14. However, we may accept the existence and position of the larger peak with confidence. The situation with regard to the smaller of the two peaks is not so clear, since it lies outside the reduced reciprocal range of the sampled frequencies. All four methods determine different values for the position of this peak, namely $\tau_{2}=1.5 \times 10^{-3}$ in Figures 14 and $16, \tau_{2}=1.4 \times 10^{-3}$ in [9], $\tau_{2}=1.2 \times 10^{-3}$ in [7] and $\tau_{2} \approx 8 \times 10^{-4}$ in [15]. The accuracy of the smaller peak can be guaranteed only if the extrapolations of the $G^{\prime}$ - and $G^{\prime \prime}$-data in Figure 16 are themselves accurate. Since extrapolation must be viewed with caution, we cannot accept the accuracy of the smaller peak with confidence, even though it provides an excellent fit to the $G^{\prime}$ - and $G^{\prime \prime}$-data in the measured range.

\section{Conclusions}

We first make some general remarks in way of summary and guidance.

Under certain conditions, a CRS can be expressed as a continuous analytical function. Under the constraint (2.3), it is always possible to express the CRS as the infinite limit of a sequence of finite series of suitable basis functions. In general, convergence to the limit can be in a least-squares sense. However, under further regularity conditions, there will always be a subsequence which converges pointwise to the CRS almost everywhere.

Throughout the paper, the parameter $N$ governs the number of terms in the wavelet expansions for $G^{\prime}$ and $G^{\prime \prime}$. There are $N+1$ terms in the expansion, the initial term being a strictly positive scaling function, and the remaining terms being wavelets of different scales. In calculating the CRS, 10th order derivatives are used in the loss algorithm and 9th order derivatives in the storage algorithm. In these algorithms, either an absolute measure of discrepancy, or a relative measure can be used. In the examples we have studied, the absolute measure is the more informative, but there is very little difference in the outcome whichever measure is adopted. 
When either algorithm is repeated, the parameter $N$, the scaling parameter $\sigma$ and the reference frequency $\omega_{0}$ should be reset. We have never needed to reset the order, $\lambda$, of the Gegenbauer wavelet. A value of $\lambda=\frac{3}{2}$ has been optimal in the examples studied. Best practice is to change one setting at a time. $N$ requires very little change. If $N$ is too large, the noise amplification will be too high, and, unless the data is exact, unphysical oscillations will emerge in the recovered spectrum. The amplification factor can only be calculated when the noise level is known in advance, as in Section 6. But the information provided in Section 6 does give a guide for the growth of the amplification factor with $\mathrm{N}$ in general. More details concerning noise amplification can be found in [1] and [2]. Varying $\sigma$ and $\omega_{0}$ is straightforward because their values are constrained. The range for $\sigma$ is given by (6.2), while the optimal $\omega_{0}$ normally resides within a decade either side of the central measured frequency. If the range of frequencies is severely limited, however, the optimal value of $\omega_{0}$ could be close to the highest measured frequency.

For noisy data, the inclusion of an estimate for $G^{\prime}(\infty)$ in the $G^{\prime}$-data set improves the statistical correlation between the two wavelet expansions for $G^{\prime}$ and $G^{\prime \prime}$. The final weighted average spectrum acts as a single model, which (i) delivers full statistical correlation between $\bar{G}^{\prime}$ and $\bar{G}^{\prime \prime}$ and (ii) generates moduli which satisfy the Kramers-Kronig relations exactly.

In this paper we have demonstrated that the link between wavelets and derivatives can facilitate the successful application of high-order derivative spectroscopy to CRS recovery. In [9], Anderssen et al make the remark "...in practice, working with experimental data, it would be prohibitively challenging to employ derivative-based formulae of order higher than 2 or 3." Whereas this remark is true in general terms, there are circumstances when the challenge is not prohibitive, as we have shown. In equations (3.3) and (3.4) the contribution of the $r$-th derivative is weighted down by a factor $(r !)^{-1}$. Thus the contribution of the 8-th derivative carries a weight of $O\left(10^{-5}\right)$ compared to the weight of the second derivative, while the 10-th derivative carries a weight of $O\left(10^{-7}\right)$ compared to the weight of the second derivative. This, combined with the properties of the specially constructed wavelet smoothing, explains why the application of high-order derivative spectroscopy is not only achievable, but is also a relatively straightforward approach to CRS recovery.

The method of wavelet regularization and other methods such as cubic spline regularization depend on a judicious choice of nodes in the numerical approximation, which can prove a lengthy computational process. One of the advantages of the approach presented in this paper is that the selection of nodal distributions is not required. The method of derivative spectroscopy described in this paper is based on exact inversion formulae for the CRS and depends on the selection of four key parameters: $N, \sigma, \omega_{0}$, and $\lambda$. Furthermore, the recovered CRS, $H_{N}$, is expressible in closed analytic form, as are the functions $\bar{G}_{N}^{\prime}$ and $\bar{G}_{N}^{\prime \prime}$ [see (6.5) and (6.6)] which reproduce the analytic curves for $G^{\prime}$ 
and $G^{\prime \prime}$ from the spectrum. We have demonstrated that the method presented in this paper is straightforward in its implementation and has advantages over other methods in the literature.

The calculations in this paper were performed on a laptop with 4GB RAM and dual-core processor operating at $1.8 \mathrm{GHz}$. The code was written in MAPLE, and CPU-time per iteration for the loss and storage algorithms varied between 5 and 10 seconds.

Acknowledgements. We are grateful for the comments on the initial manuscript received from three reviewers. These comments helped us improve the presentation of the material. We are also grateful to Scott Ankiewicz, John Dealy and Nese Orbey for useful discussions, and for providing us with the dynamic data employed in Section 6. Some of the ideas behind this work emerged during discussions in 2014 when ARD visited CSIRO Canberra. ARD is grateful for the facilities and financial assistance provided during his visit. NJG received financial support from EPSRC and from Cardiff University.

\section{References}

1. O'Haver TC, Fell AF, Smith G, Gans P, Sneddon J, Bezur L, Michel RG, Ottaway JM, Miller JN, Ahmad TA, Chadburn BP and Cottrell CT(1982). Derivative spectroscopy and its applications in analysis. Anal. Proc. 19:22-46.

2. Anderssen RS, Hegland (2010). Derivative spectroscopy - An enhanced role for numerical differentiation. J. Integ. Eqn. Appl. 22:355-367.

3. De Luca M, Oliverio F, Ioele G and Ragno G (2009). Multivariate calibration techniques applied to derivative spectroscopy data for the analysis of pharmaceutical mixtures. Chemometrics and Intelligent Laboratory Systems 96:14-21.

4. Malkin A Ya (2006). The use of a continuous relaxation spectrum for describing the viscoelastic properties of polymers. Polymer Science Series A 48:39-45.

5. Stadler FJ, Bailly C (2009). A new method for the calculation of continuous relaxation spectra from dynamic-mechanical data. Rheol Acta 48:3349.

6. Stadler F (2010). Effect of incomplete datasets on the calculation of continuous relaxation spectra from dynamic-mechanical data Rheol Acta 49:1041-1057.

7. Davies AR, Goulding NJ (2012). Wavelet regularization and the continuous relaxation spectrum. J. Non-Newtonian Fluid Mech. 189:19-30.

8. Cho KS (2013). Power series approximations of dynamic moduli and relaxation spectrum. J Rheol 57: 679-697.

9. Anderssen RS, Davies AR, de Hoog RF and Loy RJ (2015) Derivative based algorithms for continuous relaxation spectrum recovery. J NonNewtonian Fluid Mech 222:132-140. 
10. Fuoss R, Kirkwood J (1941). Electrical Properties of Solids. VIII. Dipole Moments in Polyvinyl Chloride-Diphenyl Systems. J Amer Chem Soc. 63:385-394.

11. Schwarzl F, Staverman AJ (1953). Higher approximation methods for the relaxation spectrum from static and dynamic measurements of visco-elastic materials. Appl. Sci. Res. A4:127-141.

12. Tschoegl NW (1973). A general method for the determination of approximations to the spectral distributions from the dynamic response functions. Rheol Acta 12:82-83.

13. Friedrich C (1991). A delta-function method for the $n$-th approximation of relaxation or retardation time spectrum from dynamic data. Rheol Acta 30:7-13.

14. Davies AR and Anderssen RS (1986). Improved estimates of statistical regularization parameters for Fourier differentiation and smoothing. Numer. Math. 48: 671-697.

15. Honerkamp J, Weese J (1989). Determination of the relaxation spectrum by a regularization method. Macromolecules 22:4372-4377.

16. Calderón AP (1964). Intermediate spaces and interpolation, the complex method. Stud. Math., 24:113-190.

17. Mallat S (2009). A Wavelet Tour of Signal Processing. The Sparse Way. Academic Press, San Diego, USA.

18. Fischer B and Prestin J (1997). Wavelets based on orthogonal polynomials. Math. Comp. 66:1593-1618.

19. Pathak A and Singh R Kr (2014). Solution of a system of generalized Abel integral equations using Gegenbauer wavelets. Int. J. Computer Appl. 95:1-4.

20. Abramowitz M and Stegun I (eds) (1964). Handbook of Mathematical Functions. US National Bureau of Standards, Washington DC.

21. McDougall I, Orbey N and Dealy JM (2014). Inferring meaningful relaxation spectra from experimental data. J. Rheol. 58:770-797.

22. Cho KS and Park GW (2013). Fixed-point iteration for relaxation spectrum from dynamic mechanical data. J. Rheol. 57:647-678.

23. Honerkamp J, Weese J (1993)). A nonlinear regularization method for the calculation of relaxation spectra. Rheol. Acta 32:65-73.

24. Davies AR, Anderssen RS (1997). Sampling Localization in Determining the Relaxation Spectrum. J. Non-Newtonian Fluid Mech. 73:163-179.

\section{Figures}




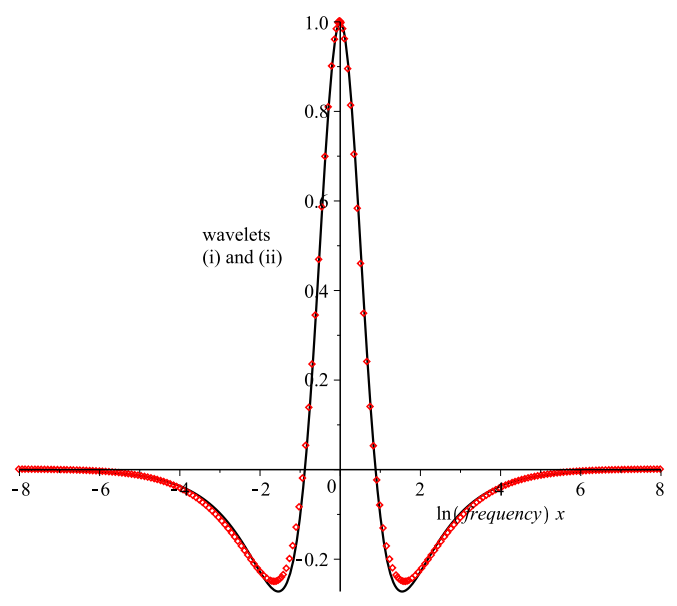

Figure 1: (i) The wavelet $-D^{2} \operatorname{sech}(\mathrm{x})($ - $)$; (ii) the wavelet $\psi^{*}\left(\frac{4}{3} x\right)$ defined by $(2.7)(\diamond \diamond \diamond)$

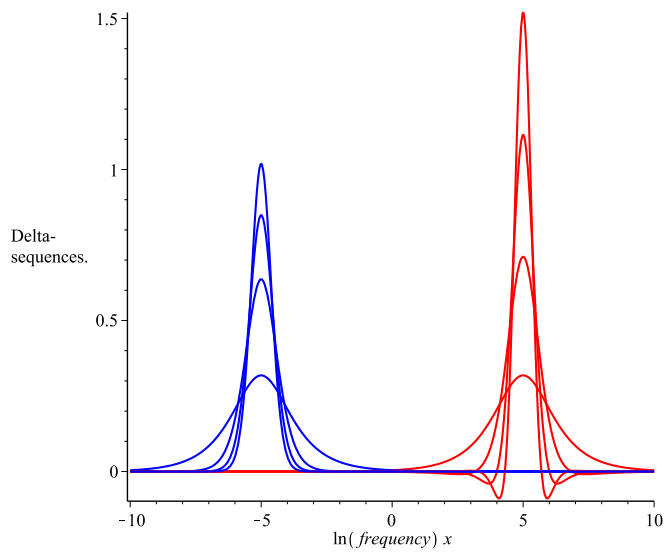

Figure 2: Left: First four even-order approximations in the Schwarzl-Staverman delta-sequence (1.8) (—); Right: First four approximations in the Maclaurin delta-sequence (3.1), $\sigma=1$ (—). 


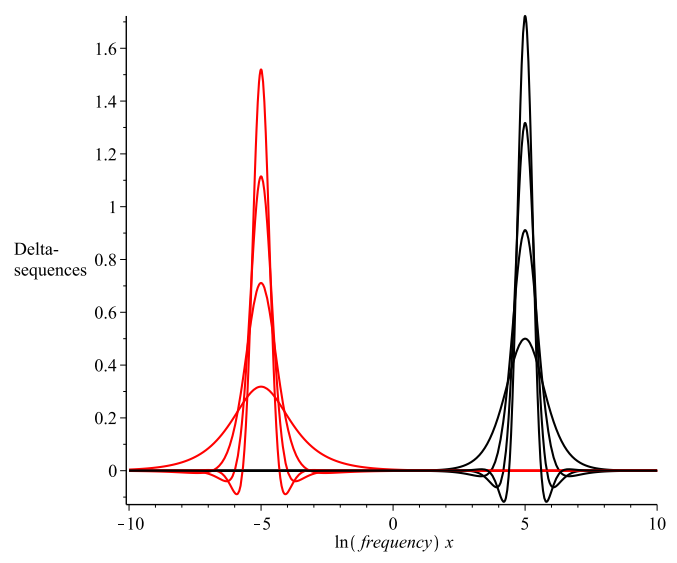

Figure 3: Left: First four approximations in the Maclaurin delta-sequence (3.1), $\sigma=1$ (—); Right: First four approximations in the Maclaurin delta-sequence (3.2), $\sigma=1$ (一).

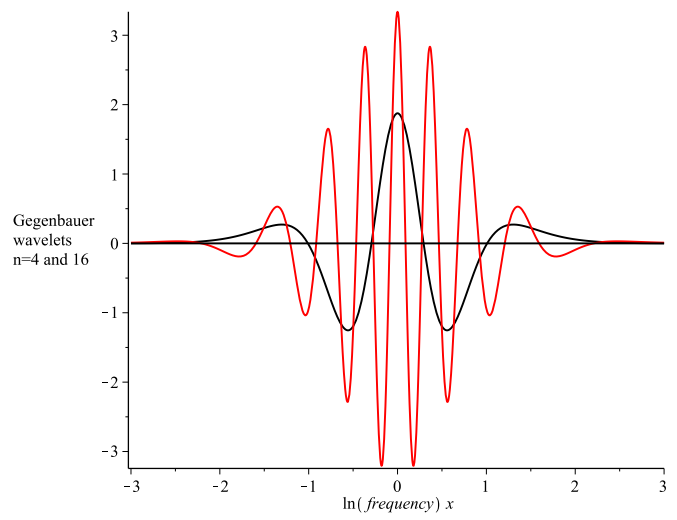

Figure 4: Two weighted Gegenbauer wavelets $\gamma_{n}^{(\lambda)}(x)$ with $\lambda=\frac{3}{2}$ : (i) $\mathrm{n}=4$ (—); (ii) $\mathrm{n}=16$ (一). 


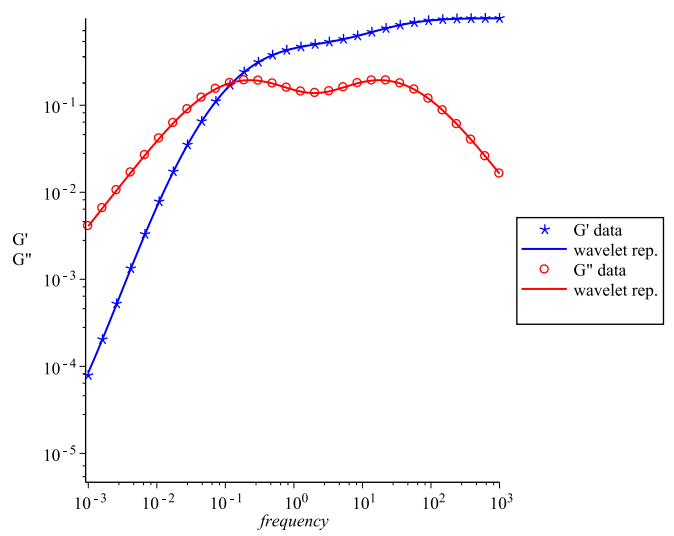

Figure 5: Precision $G^{\prime}, G^{\prime \prime}$ - data for double log-normal spectrum, with their wavelet expansion representations. $N=16, \lambda=\frac{3}{2}, \sigma=4$.

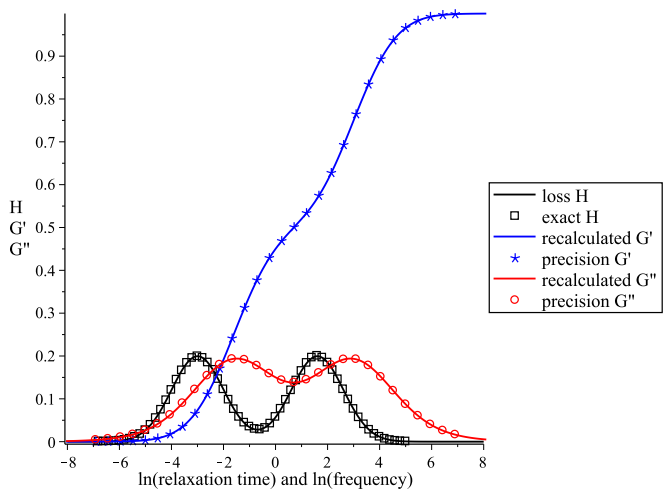

Figure 6: (i) Linear plot of exact double log-normal spectrum and its recovered loss spectrum, with $\tau=\omega^{-1}$. (ii) Precision $G^{\prime}$-data and $\bar{G}^{\prime}$ recalculated from loss spectrum. (iii) Precision $G^{\prime \prime}$-data and $\bar{G}^{\prime \prime}$ recalculated from loss spectrum. $N=16, \lambda=\frac{3}{2}, \sigma=4$. 


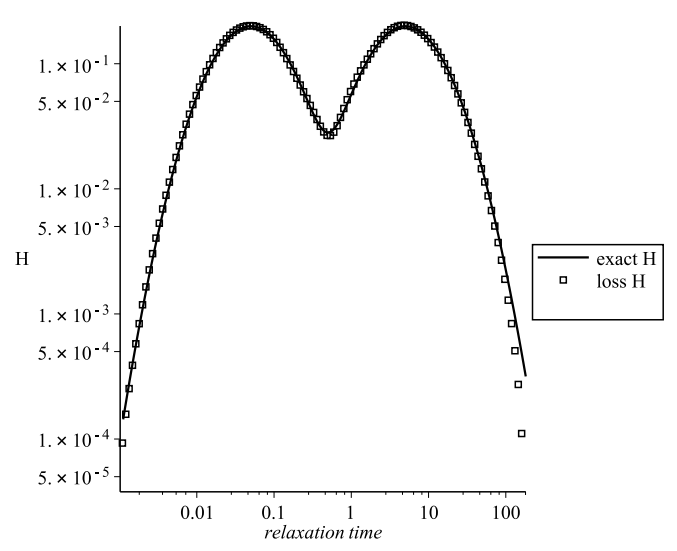

Figure 7: (i) Log-log plot of exact double log-normal spectrum and its recovered loss spectrum in Figure 6.

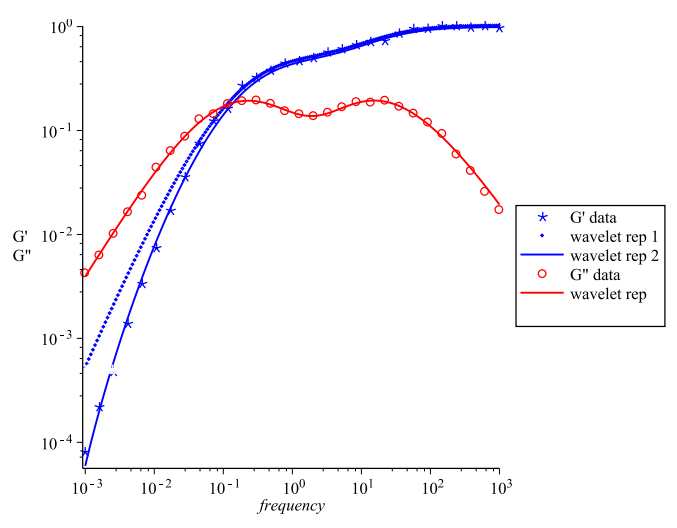

Figure 8: Noisy $G^{\prime}, G^{\prime \prime}$ - data for double log-normal spectrum, with their wavelet expansion representations. Two iterations required for $G^{\prime}$-representation. 


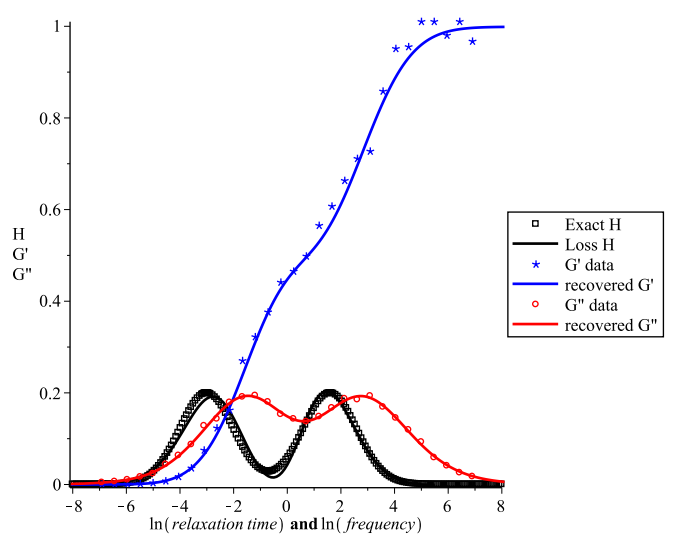

Figure 9: (i) Linear plot of exact double log-normal spectrum and its loss spectrum recovered from noisy data, with $\tau=\omega^{-1}$. (ii) Noisy $G^{\prime}$-data and $\bar{G}^{\prime}$ recalculated from loss spectrum. (iii) Noisy $G^{\prime \prime}$-data and $\bar{G}^{\prime \prime}$ recalculated from loss spectrum. $N=6, \lambda=\frac{3}{2}, \sigma=4.44$.

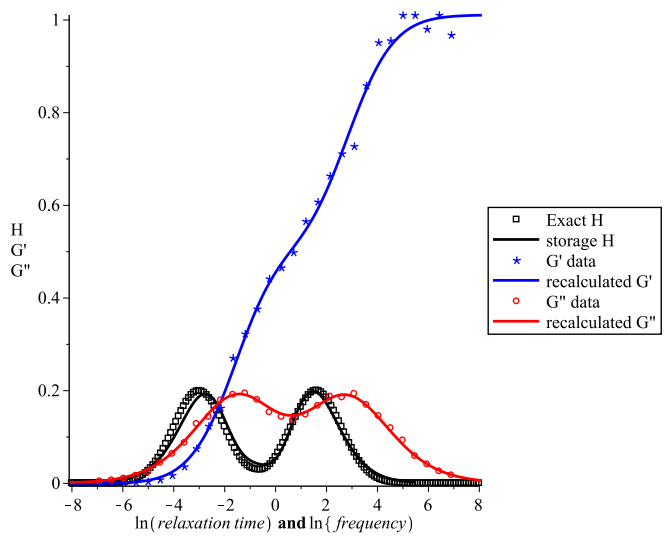

Figure 10: (i) Linear plot of exact double log-normal spectrum and its storage spectrum recovered from noisy data, with $\tau=\omega^{-1}$. (ii) Noisy $G^{\prime}$-data and $\bar{G}^{\prime}$ recalculated from storage spectrum. (iii) Noisy $G^{\prime \prime}$-data and $\bar{G}^{\prime \prime}$ recalculated from storage spectrum. $N=5, \lambda=\frac{3}{2}, \sigma=3.8$. 


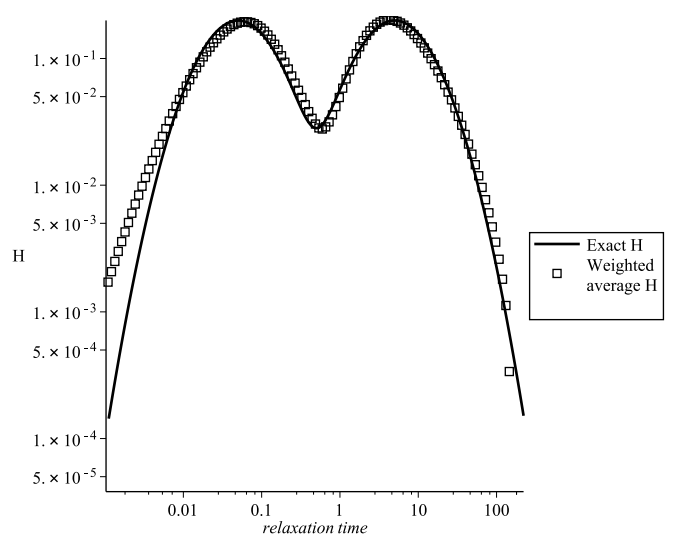

Figure 11: Log-log plot of exact double log-normal spectrum and its recovered weighted average spectrum (40\% loss, $60 \%$ storage) .

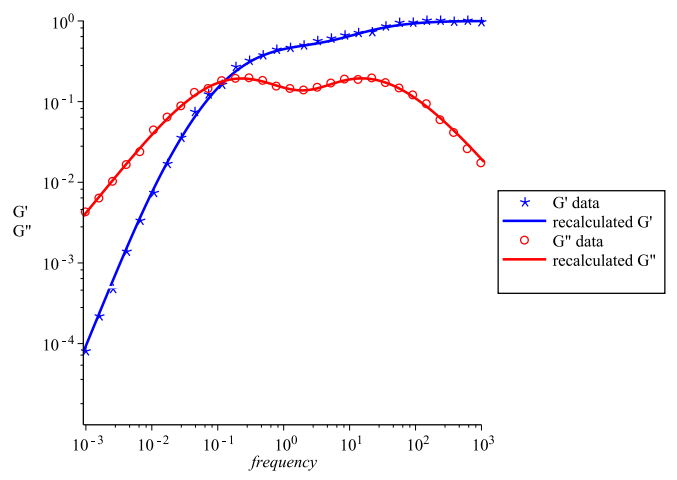

Figure 12: Noisy data for double log-normal spectrum and their recalculated moduli from weighted average spectrum in Fig 


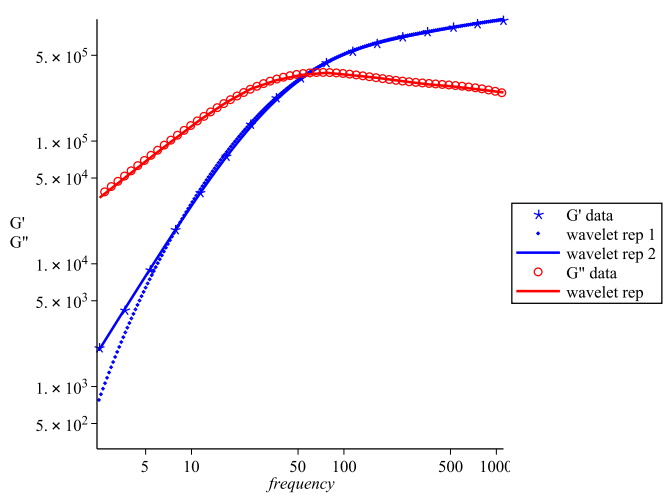

Figure 13: PBD1 $G^{\prime}, G^{\prime \prime}$ - data with their wavelet expansion representations. Two iterations required for $G^{\prime}$-representation.

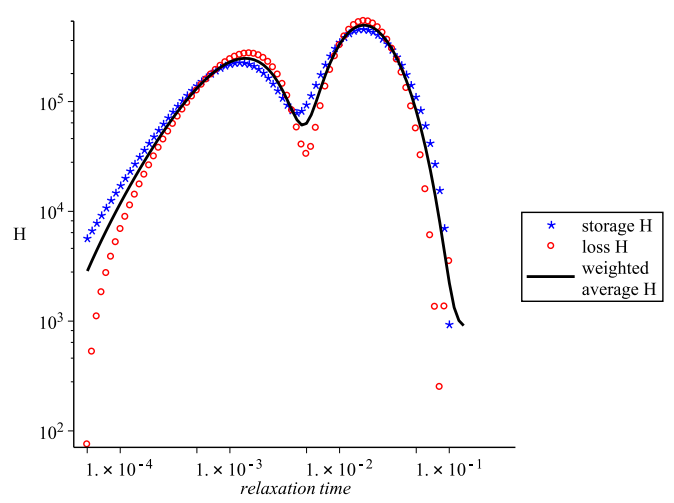

Figure 14: PBD1 data. Storage spectrum, loss spectrum and their weighted average $(45 \%$ loss, $55 \%$ storage).

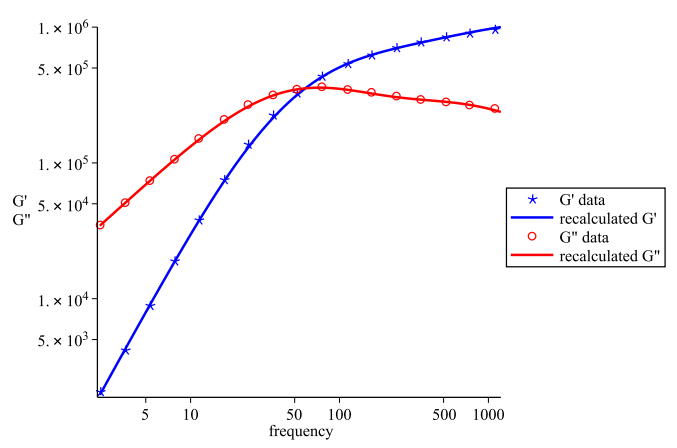

Figure 15: PBD1 data. $G^{\prime}, G^{\prime \prime}$ recalculated from weighted average spectrum in Figure 14. 


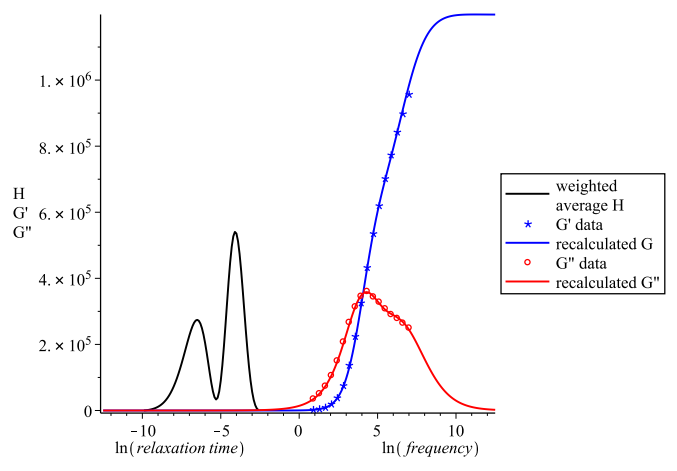

Figure 16: PBD1 data. Linear plot of weighted average spectrum in Figure 14, together with recalculated $G^{\prime}, G^{\prime \prime}$.

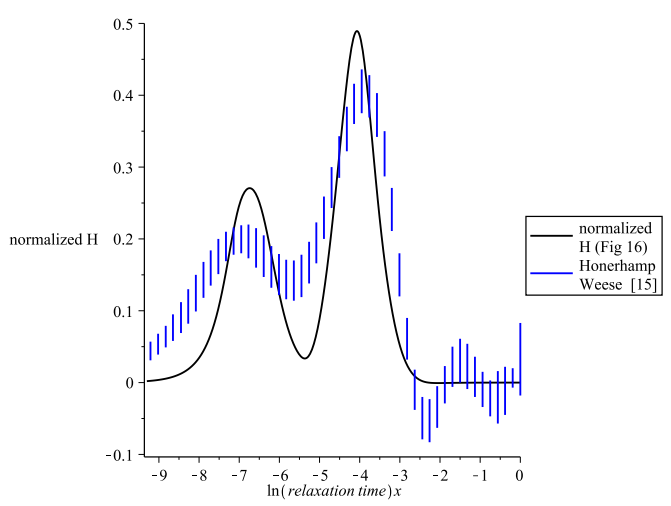

Figure 17: Normalized PBD1 spectrum from Figure 16 compared with Honerkamp \& Weese spectrum (shown with error bars), from [15]. 